\title{
Combined treatment in punctate inner choroidopathy
}

\author{
This article was published in the following Dove Press journal: \\ Therapeutics and Clinical Risk Management \\ 23 September 2016 \\ Number of times this article has been viewed
}

\author{
Barbara Terelak-Borys \\ Katarzyna Zagajewska \\ Irmina Jankowska-Lech \\ Piotr Tesla \\ Iwona Grabska-Liberek \\ Department of Ophthalmology, \\ Centre of Postgraduate Medical \\ Education, Prof W Orlowski Clinical \\ Hospital, Warsaw, Poland
}

Purpose: The purpose of this study was to describe a combination treatment for choroidal neovascular (CNV) membrane, secondary to punctate inner choroidopathy (PIC).

Patient and methods: A 44-year-old female patient was diagnosed with PIC complicated by the development of recurrent juxtafoveal neovascular membrane. The treatment included a sequence of monotherapy regimens: systemic steroid therapy, photodynamic therapy, and intravitreal injections of vascular endothelial growth factor (VEGF) inhibitor (anti-VEGF). Owing to the CNV membrane resistance to various types of monotherapy, a combination treatment consisting of local injections of steroid underneath the Tenon's capsule and intravitreal anti-VEGF injections was used.

Results: Systemic steroid therapy resulted in rapid local improvement with a very short remission period. No positive effects of photodynamic therapy were observed. Sequential anti-VEGF injections led to remission periods of several months. Permanent regression of CNV membrane was achieved following combined local application of steroid and intravitreal anti-VEGF injections.

Conclusion: A combination treatment including steroid and anti-VEGF medication characterized by anti-inflammatory and antiangiogenic effects may be a very beneficial option for the treatment of recurrent $\mathrm{CNV}$ membrane as a complication of PIC.

Keywords: punctate inner choroidopathy, neovascular membrane, anti-VEGF injections, photodynamic therapy, steroids

\section{Introduction}

Choroidal neovascularization in subfoveal locations is an important vision-threatening complication. It develops in the natural history of many ocular diseases, including inflammatory diseases of either infectious or unknown etiology. In the latter group, the complication is characterized by a very high rate of incidence in the course of punctate inner choroidopathy (PIC). ${ }^{1} \mathrm{PIC}$ is a rare inflammatory disease of the posterior ocular segment with a probably autoimmune etiology, classified as a white dot syndrome. Usually, the patients affected are young Caucasian myopic females. ${ }^{2}$ The diagnosis is made on the basis of clinical presentation. The disorder is characterized by the presence of small, circular, yellow or gray inflammatory foci within the macula as well as in the mid-periphery of retina. The lesions involve retinal pigment epithelium and deeper layers of the choroid. They are not associated with inflammation in the anterior ocular segment or the vitreous body. ${ }^{2}$ In more than half of the cases, the disease is bilateral, albeit not always simultaneous. ${ }^{2,3}$

The development of choroidal neovascular (CNV) membrane is the most serious complication of PIC. ${ }^{3}$ Subfoveal location of CNV membrane is reported very commonly in PIC in up to $100 \%$ of patients. ${ }^{1}$
Correspondence: Katarzyna Zagajewska Department of Ophthalmology, Centre of Postgraduate Medical Education, Prof W Orlowski Clinical Hospital, 23I Czerniakowska Street, 00-416 Warsaw, Poland

$\mathrm{Tel} / \mathrm{fax}+4822584 \quad$ II 85

Email katarzyna.zagajewska@gmail.com 


\section{Case presentation}

A 44-year-old female patient reported at the clinic due to impairment of vision in the right eye. Visual acuity (distance Snellen charts) was $0.5 \mathrm{cc}-0.75 \mathrm{D} \mathrm{sph} /-0.75 \mathrm{D}$ cyl, ax 170 in the right eye and 1.0 in the left eye (uncorrected). Ophthalmological examination revealed bilaterally normal anterior segments. Several lesions typical for PIC were present at the posterior pole of the right eye, without inflammation in the vitreous body. Fundoscopic image of the left eye was unremarkable. Fluorescein angiography (Figure 1) revealed a tiny CNV membrane developing from the juxtafoveal focus (Figure 1A [early phase] and B [late phase]). The patient received systemic steroid therapy, initially with methylprednisolone (intravenous 1,000 mg for 5 days) and later with prednisone (oral $0.5 \mathrm{mg} / \mathrm{kg}$ body weight $-30 \mathrm{mg} / \mathrm{d}$ initially, tapering by $5 \mathrm{mg}$ every 10 days). After 2 months of steroid treatment, best-corrected visual acuity (BCVA) of the right eye improved back to 1.0, with no features of active CNV membrane being observed clinically. One week after completion of systemic steroid therapy, BCVA was reduced to 0.6 as a result of sudden relapse and significant expansion of CNV membrane toward the fovea (Figure 1C [early phase] and D [late phase]). Photodynamic therapy (PDT) was attempted as the next treatment stage. No changes were observed in BCVA 3 months after PDT; the value remained at the level of 0.6 with further slight expansion of CNV membrane being observed angiographically (Figure 1E [early phase] and F [late phase]). Therefore, the patient was treated by intravitreal injection of vascular endothelial growth factor (VEGF) inhibitor (anti-VEGF) ranibizumab at the dose of $0.5 \mathrm{mg}$. Stabilization of visual acuity of the right eye at the level of $0.4-0.5$ was observed over the following 4 months, accompanied by regression of the CNV membrane and elimination of fluid seen in optical coherence tomography (OCT) scans (Figure 2A). Six months after the first injection, a repeated increase in CNV membrane activity confirmed by OCT led to the need of the second ranibizumab injection, again resulting in membrane regression and fluid elimination (Figure 2B [before injection] and $\mathrm{C}$ [after injection]). Eight months after the second injection, BCVA of the right eye was 0.2 (the patient failed to attend the follow-up appointments). Clinical examination, fluorescein angiography, and OCT revealed a subfoveal scar and atrophic lesions as well as recurrent $\mathrm{CNV}$ membrane growth from the original locus, this time toward the optic disk (Figures $1 \mathrm{G}$ and 2D). A combination treatment of triamcinolone at the dose of $20 \mathrm{mg}$ injected under the Tenon's capsule, followed by another intravitreal injection of ranibizumab after a 1 week interval, was administered. After 2 months, BCVA in the right eye remained at the level of 0.2 , with almost complete regression of the recurrent lesion activity (Figure 1H). Since that time, the condition has remained stable over a 5-year follow-up period (Figure 2E). The other eye remains free of pathological lesions. The patient provided oral consent to have her data used in this study.
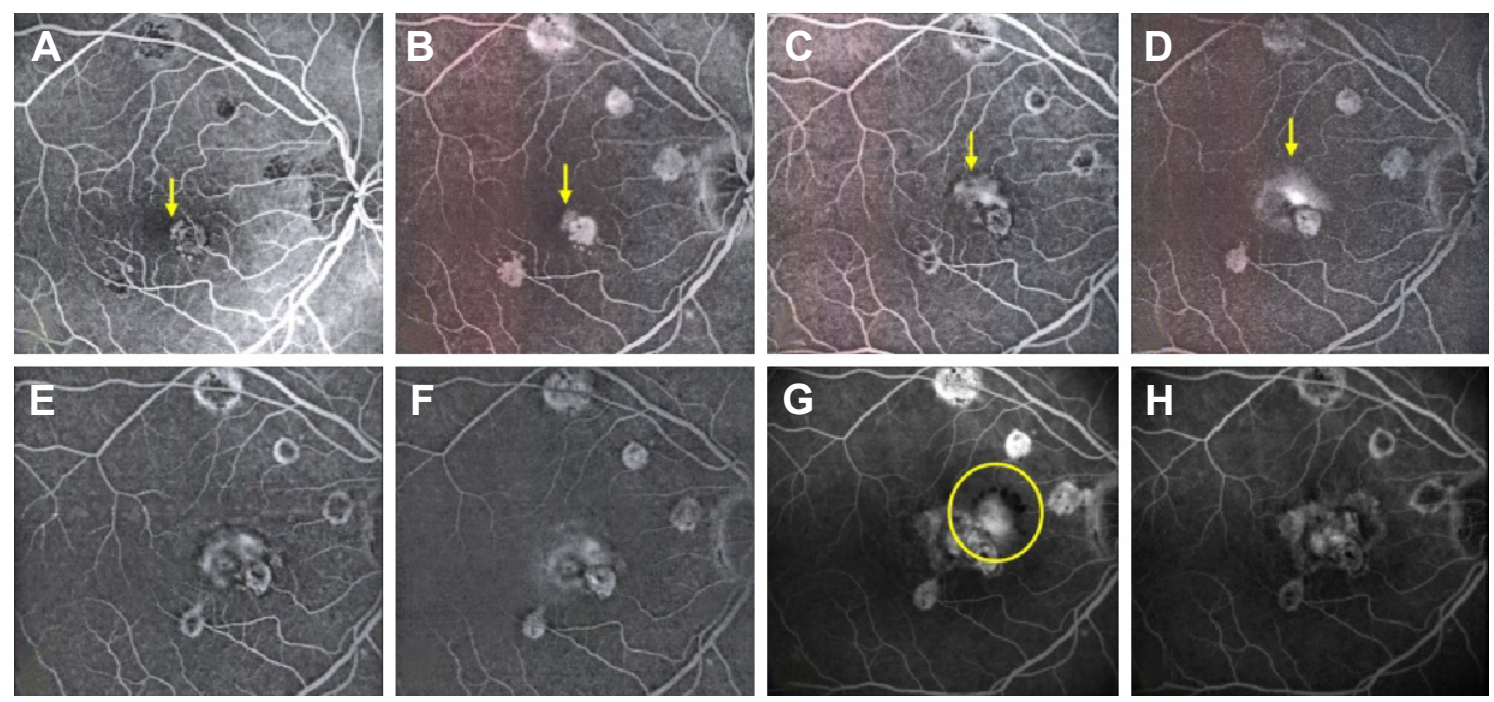

Figure I FA of the patient's right eye taken over a 2-year period.

Notes: (A and B) Initial visit (early phase of FA of the right eye reveals a small irregular hyperfluorescence area inferonasal to the fovea with late-phase leakage that corresponds to the juxtamacular CNV lesion; arrows). (C and D) One week after cessation of steroid therapy (a fulminant recurrence and development of CNV membrane with involvement of the fovea; arrows). (E and F) Three months after PDT (progressive enlargement of CNV membrane). (G) Recurrent activity of CNV membrane 8 months after the second intravitreal ranibizumab injection (circle). $(\mathbf{H})$ Two months after periocular triamcinolone injection combined with intravitreal ranibizumab injection (almost complete regression of active CNV membrane).

Abbreviations: CNV, choroidal neovascular membrane; FA, fluorescein angiography; PDT, photodynamic therapy. 

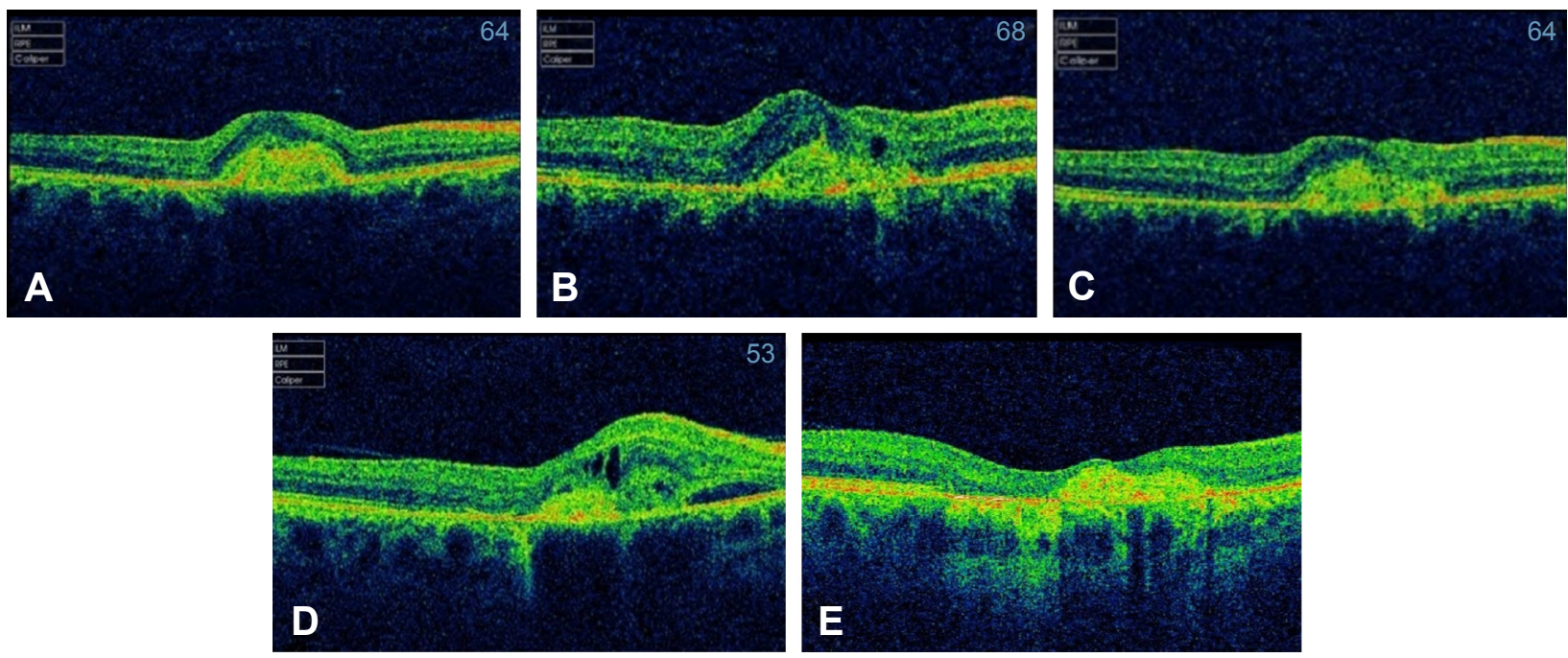

Figure 2 OCT examination results.

Notes: (A) OCT image of the right macula 2 months after the first intravitreal ranibizumab injection. Complete resolution of intraretinal fluid. (B and C) OCT images of the right macula before (B) and I month after $(\mathbf{C})$ the second intravitreal ranibizumab injection, which resulted in CNV membrane inactivation. (D) OCT images of the right macula before periocular triamcinolone injection combined with intravitreal ranibizumab injection. (E) OCT image of the right macula at 5 years. Chorioretinal atrophic changes and scarring (D).

Abbreviations: CNV, choroidal neovascular membrane; OCT, optical coherence tomography.

\section{Discussion}

No standard procedures for management of CNV membranes in the course of PIC have been established yet. Owing to the low incidence of the disorder, most cohort studies of PIC are conducted in small patient populations. The treatment of CNV membranes in the course of PIC consists of several methods, including most often systemic or local steroid therapy, PDT, and intravitreal administration of VEGF inhibitors. ${ }^{4-9}$

In the reported case, we initially resorted to intravenous and oral steroid therapy and observed rapid improvement in local condition and complete recovery of visual acuity. This result was in agreement with the findings showing that steroid therapy was particularly efficient in small CNV membranes developing as complications of inflammatory diseases of the eye fundus. ${ }^{4}$ The rapid recurrence and growth of CNV membrane within as little as 1 week from the completion of prednisone treatment was astonishing; in consequence, a decision to switch to PDT was made. PDT results in a selective angio-occlusive effect that had been initially used in the treatment of CNV membranes in the course of agerelated macular degeneration (AMD). PDT was also used in the treatment of $\mathrm{CNV}$ membranes developing as complications of inflammatory diseases of the eye fundus. ${ }^{7,10}$ In our patient, we implemented PDT after the initial steroid therapy with regard to the use of PDT in the treatment of CNV membrane secondary to inflammatory process in the course of presumed ocular histoplasmosis syndrome, ${ }^{11}$ a condition that produces a very similar clinical picture. Despite documented efficacy, PDT is associated with a risk of CNV membrane progression. ${ }^{7,10}$

Following the failure of PDT in the presented patient case, we resorted to the treatment of CNV membrane with intravitreal injection of VEGF inhibitor ranibizumab. Currently, intravitreal injections of VEGF inhibitors are used as the first-line treatment of CNV membranes in AMD. AntiVEGF agents are characterized by antiangiogenic as well as anti-inflammatory activity owing to their inhibition of excessive secretion of numerous proinflammatory cytokines such as interleukin $6{ }^{12}$ The reported results of anti-VEGF as a monotherapy of CNV membranes developing as a complication of fundus inflammatory diseases including PIC are promising, $8,9,13,14$ although cases of recurrent CNV membranes in the course of PIC were reported, requiring as many as eleven to 13 anti-VEGF injections. ${ }^{15}$ In our case, CNV membrane regression occurred after a single injection of ranibizumab and persisted for 6 months. Owing to very good response of the CNV membrane observed after the initial anti-VEGF injection, pro re nata or "as needed" treatment strategy, which is used in the long-term treatment of CNV membrane in the course of exudative AMD, was chosen as a continuation of treatment. This strategy has been chosen for the treatment of CNV membrane secondary to PIC in other studies. ${ }^{8}$ Another injection was delivered following reactivation of $\mathrm{CNV}$ membrane, also with a positive outcome. Owing to CNV membrane recurrence following the second 
injection, the possibility of a combination treatment was taken into consideration. The available literature contains reports of the efficacy of combinations including PDT with systemic steroid therapy, ${ }^{16}$ as well as PDT with intravitreal triamcinolone. ${ }^{17}$ The reported results of combined administration of PDT and anti-VEGF medications were encouraging, albeit also requiring multiple injections. ${ }^{9}$

Owing to the adverse effect of PDT consisting of enhanced VEGF production, ${ }^{18}$ possibly leading to $\mathrm{CNV}$ membrane progression despite the angio-occlusive effect of PDT, that was also observed in this case, we decided to continue with the combination treatment involving local periocular administration of a steroid under the capsule of Tenon and intravitreal injection of an anti-VEGF agent. The beneficial effects of steroid and anti-VEGF agents had been previously observed in the patient. Owing to extrafoveal location of reactivated CNV membrane, not threatening fixation, we decided to use a less burdening local steroid therapy administered as periocular injection to avoid the adverse systemic effects of steroid therapy as well as side effects that often accompany intravitreal administration of steroids (cataract, increased intraocular pressure). Single administration of a combination treatment allowed suppression of CNV membrane recurrence over a 5-year follow-up period. We found no reports on the use of this type of combination treatment in the available literature.

\section{Conclusion}

To sum up, based on the observation of the presented patient case, it appears that the combined use of steroid and antiVEGF medications characterized by anti-inflammatory and antiangiogenic effects may be a very beneficial option for the treatment of recurrent CNV membrane as a complication of PIC. Since this is a single-case report, the conclusion from this study is limited and further investigations to confirm our results are needed.

It should be stressed that the conclusions assessing the efficacy of individual treatment methods in PIC are based only on individual case reports or on small series of cases. PIC is a rare disorder; thus, a reliable evaluation of the efficacy of treatment methods is difficult to establish.

\section{Author contributions}

All authors contributed toward data analysis, drafting, and critically revising the paper and agree to be accountable for all aspects of the work.

\section{Disclosure}

The authors report no conflicts of interest in this work.

\section{References}

1. Kedhar SR, Thorne JE, Wittenberg S, Dunn JP, Jabs DA. Multifocal choroiditis with panuveitis and punctate inner choroidopathy: comparison of clinical characteristics at presentation. Retina. 2007;27(9): 1174-1179.

2. Watzke RC, Packer AJ, Folk JC, Benson WE, Burgess D, Ober RR. Punctate inner choroidopathy. Am J Ophthalmol. 1984;98(5):572-584.

3. Patel KH, Birnbaum AD, Tessler HH, Goldstein DA. Presentation and outcome of patients with punctate inner choroidopathy at a tertiary referral center. Retina. 2011;31(7):1387-1391.

4. Brown J Jr, Folk JC, Reddy CV, Kimura AE. Visual prognosis of multifocal choroiditis, punctate inner choroidopathy, and the diffuse subretinal syndrome. Ophthalmology. 1996;103(7):1100-1105.

5. Flaxel CJ, Owens SL, Mulholland B, Schwartz SD, Gregor ZJ. The use of corticosteroids for choroidal neovascularisation in young patients. Eye (Lond). 1998;12(pt 2):266-272.

6. Sim DA, Sheth HG, Kaines A, Tufail A. Punctate inner choroidopathyassociated choroidal neovascular membranes during pregnancy. Eye (Lond). 2008;22(5):725-727.

7. Wachtlin J, Heimann H, Behme T, Foerster MH. Long-term results after photodynamic therapy with verteporfin for choroidal neovascularizations secondary to inflammatory chorioretinal diseases. Graefes Arch Clin Exp Ophthalmol. 2003;241(11):899-906.

8. Menezo V, Cuthbertson F, Downes SM. Positive response to intravitreal ranibizumab in the treatment of choroidal neovascularization secondary to punctate inner choroidopathy. Retina. 2010;30(9):1400-1404.

9. Cornish KS, Williams GJ, Gavin MP, Imrie FR. Visual and optical coherence tomography outcomes of intravitreal bevacizumab and ranibizumab in inflammatory choroidal neovascularization secondary to punctate inner choroidopathy. Eur J Ophthalmol. 2011;21(4): 440-445.

10. Postelmans L, Pasteels B, Coquelet P, et al. Photodynamic therapy for subfoveal classic choroidal neovascularization related to punctate inner choroidopathy (PIC) or presumed ocular histoplasmosis-like syndrome (POHS-like). Ocul Immunol Inflamm. 2005;13(5):361-366.

11. Saperstein DA, Rosenfeld PJ, Bressler NM, et al; Verteporfin in Ocular Histoplasmosis ( $\mathrm{VOH})$ Study Group. Photodynamic therapy of subfoveal choroidal neovascularization with verteporfin in the ocular histoplasmosis syndrome: one-year results of an uncontrolled, prospective case series. Ophthalmology. 2002;109(8):1499-1505.

12. Nolan A, Weiden MD, Thurston G, Gold JA. Vascular endothelial growth factor blockade reduces plasma cytokines in a murine model of polymicrobial sepsis. Inflammation. 2004;28(5):271-278.

13. Chan WM, Lai TY, Liu DT, Lam DS. Intravitreal bevacizumab (Avastin) for choroidal neovascularization secondary to central serous chorioretinopathy, secondary to punctate inner choroidopathy, or of idiopathic origin. Am J Ophthalmol. 2007;143(6):977-983.

14. Rouvas A, Petrou P, Douvali M, et al. Intravitreal ranibizumab for the treatment of inflammatory choroidal neovascularization. Retina. 2011;31(5):871-879.

15. Mangat SS, Ramasamy B, Prasad S, Walters G, Mohammed M, Mckibbin M. Resolution of choroidal neovascularization secondary to punctate inner choroidopathy (PIC) with intravitreal anti-VEGF agents: a case series. Semin Ophthalmol. 2011;26(1):1-3.

16. Fong KC, Thomas D, Amin K, Inzerillo D, Horgan SE. Photodynamic therapy combined with systemic corticosteroids for choroidal neovascularisation secondary to punctate inner choroidopathy. Eye (Lond). 2008; 22(4):528-533.

17. Chan WM, Lai TY, Lau TT, Lee VY, Liu DT, Lam DS. Combined photodynamic therapy and intravitreal triamcinolone for choroidal neovascularization secondary to punctate inner choroidopathy or of idiopathic origin: one-year results of a prospective series. Retina. 2008; 28(1):71-80.

18. Schmidt-Erfurth U, Schlötzer-Schrehard U, Cursiefen C, Michels S, Beckendorf A, Naumann GO. Influence of photodynamic therapy on expression of vascular endothelial growth factor (VEGF), VEGF receptor 3, and pigment epithelium-derived factor. Invest Ophthalmol Vis Sci. 2003;44(10):4473-4480. 
Therapeutics and Clinical Risk Management

Dovepress

\section{Publish your work in this journal}

Therapeutics and Clinical Risk Management is an international, peerreviewed journal of clinical therapeutics and risk management, focusing on concise rapid reporting of clinical studies in all therapeutic areas, outcomes, safety, and programs for the effective, safe, and sustained use of medicines. This journal is indexed on PubMed Central, CAS,

EMBase, Scopus and the Elsevier Bibliographic databases. The manuscript management system is completely online and includes a very quick and fair peer-review system, which is all easy to use. Visit http://www.dovepress.com/testimonials.php to read real quotes from published authors.

Submit your manuscript here: http://www.dovepress.com/therapeutics-and-clinical-risk-management-journal 